\title{
Erratum to: Modeling State-Conditional Observation Distribution Using Weighted Stereo Samples for Factorial Speech Processing Models
}

\author{
Mahdi Khademian' ${ }^{1}$. \\ Mohammad Mehdi Homayounpour ${ }^{1}$
}

\section{Erratum to: Circuits Syst Signal Process DOI 10.1007/s00034-016-0310-y}

This article has been updated from its original publication. Text in the first full paragraph after Eq. 23 was updated to read: Comparing WSS empirical distribution to empirical distribution of IDPMC, we can observe that while the extracted samples in IDPMC are equally weighted, they are extracted using the approximated mismatch functions.

This has been corrected in the original version of the article.

The online version of the original article can be found under doi:10.1007/s00034-016-0310-y.

Mohammad Mehdi Homayounpour homayoun@aut.ac.ir

Mahdi Khademian

khademian@aut.ac.ir

1 Laboratory for Intelligent Multimedia Processing (LIMP), Amirkabir University of Technology, Tehran, Iran 